\title{
Anti-inflammatory effects of BHBA in both in vivo and in vitro Parkinson's disease models are mediated by GPR109A-dependent mechanisms
}

Shou-Peng Fu ${ }^{1 \dagger}$, Jian-Fa Wang ${ }^{2 \dagger}$, Wen-Jing Xue ${ }^{1 \dagger}$, Hong-Mei Liu ${ }^{1 \dagger}$, Bing-run Liu ${ }^{1 \dagger}$, Ya-Long Zeng ${ }^{1}$, Su-Nan Li ${ }^{1}$, Bing-Xu Huang ${ }^{1}$, Qing-Kang LV', Wei Wang ${ }^{1,2^{*}}$ and Ju-Xiong Liu ${ }^{1 *}$

\begin{abstract}
Background: Accumulating evidence suggests that neuroinflammation plays an important role in the progression of Parkinson's disease (PD). Excessively activated microglia produce several pro-inflammatory enzymes and pro-inflammatory cytokines, leading to damage to surrounding neurons and eventually inducing neurodegeneration. Therefore, the inhibition of microglial overactivation may be a potential therapeutic strategy to prevent the further progression of PD. $\beta$-Hydroxybutyric acid (BHBA) has been shown to suppress lipopolysaccharide (LPS)-induced inflammation in BV-2 cells and to protect dopaminergic neurons in previous studies, but the underlying mechanisms remain unclear. Thus, in this study, we further investigated this mechanism in LPS-induced in vivo and in vitro PD models.

Methods: For the in vitro experiments, primary mesencephalic neuron-glia cultures were pretreated with BHBA and stimulated with LPS. $\left.{ }^{3} \mathrm{H}\right]$ dopamine (DA) uptake, tyrosine hydroxylase-immunoreactive (TH-ir) neurons and morphological analysis were evaluated and analyzed in primary mesencephalic neuron-glia cultures. In vivo, microglial activation and the injury of dopaminergic neurons were induced by LPS intranigral injection, and the effects of BHBA treatment on microglial activation and the survival ratio and function of dopaminergic neurons were investigated. Four our in vitro mechanistic experiment, primary microglial cells were pretreated with BHBA and stimulated with LPS; the cells were then assessed for the responses of pro-inflammatory enzymes and pro-inflammatory cytokines, and the NF-KB signaling pathway was evaluated and analyzed.
\end{abstract}

Results: We found that BHBA concentration-dependently attenuated the LPS-induced decrease in $\left.{ }^{3} \mathrm{H}\right] \mathrm{DA}$ uptake and loss of TH-ir neurons in the primary mesencephalic neuron/glia mixed culture. BHBA treatment significantly improved the motor dysfunction of the PD model rats induced by intranigral injection of LPS, and this beneficial effect of BHBA was attributed to the inhibition of microglial overactivation and the protection of dopaminergic neurons in the substantia nigra (SN). Our in vitro mechanistic study revealed that the inhibitory effect of BHBA on microglia was mediated by G-protein-coupled receptor 109A (GPR109A) and involved the NF-kB signaling pathway, causing the inhibition of pro-inflammatory enzyme (iNOS and COX-2) and pro-inflammatory cytokine (TNF-a, IL-1 $\beta$, and IL-6) production.

Conclusions: In conclusion, the present study supports the effectiveness of BHBA in protecting dopaminergic neurons against inflammatory challenge.

Keywords: BHBA, GPR109A, Parkinson's disease, neuroinflammation, LPS, NF-kB

\footnotetext{
* Correspondence: wangwei@jluhp.edu.cn; juxiong@jlu.edu.cn

${ }^{\dagger}$ Equal contributors

${ }^{1}$ College of Veterinary Medicine Jilin University, Changchun 130062, P R

China

${ }^{2}$ College of Animal Science and Veterinary Medicine, Heilongjiang Bayi

Agricultural University, Daqing 163319, P R China
} 


\section{Background}

Parkinson's disease (PD) is the second most prevalent neurodegenerative disorder, affecting millions of people worldwide [1]. A major hallmark of PD is the loss of dopaminergic neurons in the substantia nigra par compacta (SNpc) of the midbrain [2]. The loss of dopaminergic neurons in PD leads to motor dysfunction accompanied by progressive non-motor symptoms, which include cognitive impairments, mood disturbances, sleep dysfunction, gastrointestinal problems, and dysautonomia [3-5]. Although the exact mechanisms underlying PD pathogenesis are yet to be defined, oxidative stress, mitochondrial dysfunction, and inflammation may contribute to this process [6-8].

Accumulating evidence suggests that neuroinflammation plays an important role in the progression of $\mathrm{PD}[9,10]$. Post-mortem studies have shown that there is a large number of reactive microglia in the substantia nigra (SN) in $\mathrm{PD}$, particularly in areas of maximal neurodegeneration, namely the ventral and lateral regions of the SN [11]. A robust activation of microglia has also been found in both 1-methyl-4-phenyl-1, 2, 3, 6-tetrahydropyridine (MPTP)- and 6-hydroxydopamine (6-OHDA)-induced PD animal models $[12,13]$. Uncontrolled overactivation of microglia is a major component of neuroinflammation. Excessive activation of microglia and the consequent release of several pro-inflammatory cytokines and/or pro-inflammatory enzymes, such as TNF- $\alpha$, IL-1 $\beta$, IL-6, inducible nitric oxide synthase (iNOS), and cyclooxygenase-2 (COX-2), are believed to contribute to neurodegenerative processes $[14,15]$. Therefore, inhibition of microglial overactivation may be a potential therapeutic strategy to prevent further progression of PD.

In mesencephalic neuron-glia cultures, the stimulation of microglia with inflammagen lipopolysaccharide (LPS) induces the production of factors, including TNF- $\alpha$, IL- $1 \beta$, IL-6, iNOS, and COX-2 [16,17]. Studies have attributed the accumulation of these factors to the degeneration of dopaminergic neurons [18-20]. The intranigral infusion of LPS in rats results in the significant degeneration of nigral dopaminergic neurons and depletion of striatal dopamine (DA) $[21,22]$. Therefore, these in vitro and in vivo models of inflammation-mediated dopaminergic neurodegeneration are powerful tools in mechanistic studies and the identification of potential therapeutic agents.

$\beta$-Hydroxybutyric acid (BHBA) is an important intermediate of amino and fatty acid catabolism that has been demonstrated to be neuroprotective [23,24]. Previous studies have shown that BHBA has strong protective effects in an MPTP-induced PD mouse model [25] and provides substantial protection against apoptosis of dopaminergic neurons intoxicated by 1-methyl-4-phenylpyri-dinium $\left(\mathrm{MPP}^{+}\right)$[24], demonstrating that it is a potent neuroprotectant in both in vivo and in vitro PD models. Previous mechanistic studies have revealed that the anti-inflammatory effects of BHBA contributed to its neuroprotective effects $[15,26]$, but the precise underlying mechanism is still unclear. The purpose of the present study was to investigate the neuroprotective and anti-inflammatory properties of BHBA in LPS-induced in vivo and in vitro PD models and to identify the specific anti-inflammatory mechanism of BHBA.

\section{Methods}

\section{Animals and surgery}

Male Wistar rats (250 to 290 g) were obtained from the Center of Experimental Animals of the Baiqiuen Medical College of Jilin University (Jilin, China). The rats were maintained in plastic cages under conventional conditions. Water and pelleted diets were supplied ad libitum. Studies were performed in accordance with the guidelines established by the Jilin University Institutional Animal Care and Use Committee. The animals were allowed to acclimate to their new surroundings for 7 days before experimental manipulations. They were anesthetized with sodium pentobarbital (45 mg/kg, i.p.) and positioned in a stereotaxic apparatus (David Kopf Instruments, Tujunga, CA, U.S.A) to conform to the brain atlas of Paxinos and Watson [27]. LPS (obtained from Escherichia coli, serotype O26:B6; Sigma-Aldrich, St. Louis, MO, USA) were dissolved $(5 \mathrm{mg} / \mathrm{ml})$ in phosphate-buffed saline (PBS), and $2.0 \mu \mathrm{l}$ was injected into the right $\mathrm{SNpc}$ at a rate of $0.2 \mu \mathrm{l} / \mathrm{min}$. The injection needle was lowered through a drill hole at $5.3 \mathrm{~mm}$ posterior, $2 \mathrm{~mm}$ lateral, and $7.8 \mathrm{~mm}$ ventral to the bregma. The injections were delivered over a period of approximately $10 \mathrm{~min}$. Then, the needle was left in situ for $5 \mathrm{~min}$ to avoid reflux along the injection track. Thereafter, the skull surface was covered with fibrosponge, and the skin was sutured. Sham-operated animals were subjected to the same surgical procedures, except that $2 \mu \mathrm{l}$ of PBS was injected into the SNpc.

\section{Application of $\beta$-hydroxybutyric acid}

Rats were divided into the following five groups: the sham-operated group, the LPS-injected group followed by vehicle treatment, and the LPS-injected group followed by treatment with $0.4,0.8$, or $1.6 \mathrm{mmol} / \mathrm{kg} / \mathrm{d}$ BHBA (Sigma-Aldrich, St. Louis, MO, USA). BHBA was resolved in PBS and administered subcutaneously $(1 \mu \mathrm{l} / \mathrm{h})$ using Alzet mini-osmotic pumps (DURECT Corp., Cupertino, California, CA, USA). The rats received BHBA from 3 days before LPS injection up to 21 days post-LPS injection (24 days in total).

\section{Rotational behavior assay}

A rotational behavior assay was performed according to a previously described protocol [21,22]. Briefly, rats were placed into cylinders attached to a rotameter (Columbus Instruments, Columbus, OH, USA) and allowed to adapt 
for $10 \mathrm{~min}$ to the testing environment. Then, they were intraperitoneally injected with $5 \mathrm{mg} / \mathrm{kg} \mathrm{D}$-amphetamine sulfate (Sigma-Aldrich, St. Louis, MO, USA) dissolved in physiological saline. Measurements of rotational activity began at $5 \mathrm{~min}$ after injection and lasted for $30 \mathrm{~min}$ under minimal external stimuli. The number of turns made during the entire 30-min testing period was counted.

\section{Rat mesencephalic neuron-glia cultures}

Embryonic mesencephalic neuron-glia cultures were obtained from timed-pregnant Wistar rats on embryonic day 14. Briefly, ventral mesencephalic tissues were removed and dissociated to single cells by a mechano-enzymatic method involving a protease treatment with $2.5 \mathrm{mg} / \mathrm{ml}$ trypsin and $0.1 \mathrm{mg} / \mathrm{ml}$ DNAse type I (Sigma-Aldrich, St. Louis, MO, USA) and additional mechanical shearing. Cells were seeded at $2 \times 10^{5}$ per well in 24-well culture plates precoated with poly-D-lysine $(1 \mathrm{mg} / \mathrm{ml})$ (Sigma-Aldrich, St. Louis, MO, USA) and maintained at $37^{\circ} \mathrm{C}$ in a humidified atmosphere of $5 \% \mathrm{CO}_{2}$ and $95 \%$ air in a maintenance medium consisting of minimum essential medium supplemented with $10 \%$ heat-inactivated fetal bovine serum and $10 \%$ heat-inactivated horse serum (Gibco Life Technologies, Inc., Grand Island, NY), 2 mM L-glutamine, $1 \mathrm{mM}$ sodium pyruvate, $100 \mu \mathrm{M}$ nonessential amino acids, $50 \mathrm{U} / \mathrm{ml}$ penicillin, and $50 \mu \mathrm{g} / \mathrm{ml}$ streptomycin (Gibco Life Technologies, Inc., Grand Island, NY). Seven-day-old cultures were used for the treatment.

\section{Primary microglia-enriched cultures}

Rat microglia-enriched cultures were prepared according to a previously described protocol $[28,29]$. Briefly, whole brains of 1-day-old neonatal Wistar rats, with the blood vessels and meninges removed, were triturated in Hank's balanced salt solution. Cells $\left(2.5 \times 10^{7}\right)$ were seeded in $150-\mathrm{cm}^{2}$ culture flasks in $15 \mathrm{ml}$ of a Dulbecco's modified Eagle's medium/nutrient mixture F12 mixture (1:1) (Gibco Life Technologies, Inc., Grand Island, NY) containing 10\% heat-inactivated FBS, 2 mM L-glutamine, $1 \mathrm{mM}$ sodium pyruvate, $100 \mu \mathrm{M}$ nonessential amino acids, $50 \mathrm{U} / \mathrm{ml} \mathrm{penicillin,} \mathrm{and} 50 \mu \mathrm{g} / \mathrm{ml}$ streptomycin. The cultures were maintained at $37^{\circ} \mathrm{C}$ in a humidified atmosphere of $5 \% \quad \mathrm{CO}_{2}$ and $95 \%$ air. The medium (15 $\mathrm{ml} /$ flask) was replenished at 1 and 4 days after the initial seeding and changed every third day thereafter. Upon reaching confluence (day 14), the microglia were shaken off (200 rpm for $4 \mathrm{~h}$ on an orbital shaker), pelleted at $800 \mathrm{~g}$ for $10 \mathrm{~min}$, resuspended in fresh medium, and plated $\left(10^{5}\right.$ cells/well) in 24-well culture plates. Twenty-four hours later, the cells were ready for treatment. The purity of the microglial culture was $>98 \%$ as previously determined by immunofluorescence and cytochemical analysis [30].

\section{$\left[{ }^{3} \mathrm{H}\right] \mathrm{DA}$ uptake assay}

Cultures were incubated for $20 \mathrm{~min}$ at $37^{\circ} \mathrm{C}$ with $1 \mu \mathrm{M}$ $\left[{ }^{3} \mathrm{H}\right]$ dopamine (DA) in Krebs-Ringer buffer (SigmaAldrich, St. Louis, MO, USA). After washing three times with ice-cold Krebs-Ringer buffer, the cells were lysed in $1 \mathrm{~N} \mathrm{NaOH}$. A liquid scintillation counter (Tri-Carb, model 3314, Packard) was used for measuring radioactivity. Nonspecific DA uptake observed in the presence of mazindol $(10 \mu \mathrm{M})$ was subtracted.

\section{High-performance liquid chromatography}

High-performance liquid chromatography (HPLC) analysis was performed according to a previously described protocol for DA and its metabolite 3,4-dihydroxyphenylacetic acid (DOPAC) $[21,22]$. Briefly, SNs were weighed and suspended in $200 \mathrm{mM}$ ice-cold perchloric acid. Each sample was sonicated and then placed in an ice bath for $60 \mathrm{~min}$. Subsequently, the sample was centrifuged at 20,000 g for $20 \mathrm{~min}$ at $4^{\circ} \mathrm{C}$. The supernatant was transferred to a clean tube, and the volume was measured. Onehalf volume of a potassium dihydrogen phosphate solution was added to the supernatant and centrifuged at 20,000 $\mathrm{g}$ for $20 \mathrm{~min}$ at $4^{\circ} \mathrm{C}$. An aliquot of the supernatant was injected into an HPLC system for analysis.

\section{RNA interference}

G-protein-coupled receptor 109A (GPR109A) siRNAs were purchased from OriGene (OriGene Technologies, Beijing, China) and complexed with Lipofectamine 2000 (Invitrogen, Carlsbad, CA, USA) in 24-well plates, according to the manufacturer's instructions.

\section{RNA extraction, reverse transcription and quantitative real-time PCR}

Total RNA was extracted from the cells using Trizol (Invitrogen, Carlsbad, CA, USA), according to the supplier's protocol. Total RNA was then treated with RNase-free Dnase I, quantified by measuring the absorbance at 260 and $280 \mathrm{~nm}$ and stored at $-80^{\circ} \mathrm{C}$ until analysis. The extracted RNA was subjected to RT-PCR using a PrimeScript RT Reagent Kit With gDNA Eraser (Takara Shuzo Co., Ltd., Kyoto, Japan). The mRNA levels of various genes were evaluated by quantitative polymerase chain reaction (qRT-PCR) using a SYBR Green QuantiTect RT-PCR Kit (Roche, South San Francisco, CA, USA), and each sample was assessed in triplicate. The relative expression levels of iNOS, COX-2, TNF- $\alpha$, IL-1 $\beta$, IL-6, and GPR109A were calculated relative to $\beta$-actin (the normalizer) using the comparative cycle threshold method. The primer sequences for the tested genes are shown in Table 1. 
Table 1 The primer sequences of $\beta$-actin, GPR109A, iNOS, COX-2, TNF- $\alpha$, IL-1 $\beta$, and IL-6

\begin{tabular}{|c|c|c|}
\hline Gene & Sequences & Length (bp) \\
\hline \multirow[t]{2}{*}{$\beta$-actin } & (F) 5'- GTCAGGTCATCACTATCGGCAAT -3' & 147 \\
\hline & (R) 5'- AGAGGTCTTTACGGATGTCAACGT -3' & \\
\hline \multirow[t]{2}{*}{ GPR109A } & (F) 5'- GCTGCCCTGTCGGTTCAT -3' & 134 \\
\hline & (R) 5'- CGTGGCTGACTTTCTCCTGAT -3' & \\
\hline \multirow[t]{2}{*}{ iNOS } & (F) 5'- CACCCAGAAGAGTTACAGC -3' & 186 \\
\hline & (R) 5'- GGAGGGAAGGGAGAATAG -3' & \\
\hline \multirow[t]{2}{*}{$\operatorname{cox}-2$} & (F) 5'- AGAGTCAGTTAGTGGGTAGT -3' & 170 \\
\hline & (R) 5'- CTTGTAGTAGGCTTAAACATAG -3' & \\
\hline \multirow[t]{2}{*}{ TNF-a } & (F) 5'- CCACGCTCTTCTGTCTACTG -3' & 145 \\
\hline & (R) 5'- GCTACGGGCTTGTCACTC -3' & \\
\hline \multirow[t]{2}{*}{$\mid L-1 \beta$} & (F) 5'- TGTGATGTTCCCATTAGAC -3' & 131 \\
\hline & (R) 5'- AATACCACTTGTTGGCTTA -3' & \\
\hline \multirow[t]{2}{*}{ IL-6 } & (F) 5'- AGCCACTGCCTTCCCTAC -3' & 156 \\
\hline & (R) 5'- TTGCCATTGCACAACTCTT -3' & \\
\hline
\end{tabular}

\section{ELISA}

The amounts of TNF- $\alpha$, IL- $1 \beta$, and IL- 6 in the culture medium were measured with commercial ELISA kits obtained from BioLegend.

\section{Tyrosine hydroxylase and IBA-1 immunohistological analysis}

The brains were fixed and processed for immunostaining as described previously [31]. The primary antibodies used in this study were as follows: rabbit polyclonal anti-tyrosine hydroxylase (TH) (1:1000; Abcam, Cambridge, CA, USA) and ionized calcium-binding adaptor molecule-1 (IBA-1) (1:200, Proteintech, Chicago, IL, USA). To determine cell numbers, total nigral TH-positive cells were counted by three researchers blind to the experimental design, and the average of these scores were reported.

\section{Western blot analysis}

After the last behavioral test, the SNs of the rats were rapidly dissected out, frozen, and stored in a deep freezer at $-80^{\circ} \mathrm{C}$ until the assays. The rat brain $\mathrm{SNs}$ and the microglial cells were lysed in lysis buffer (Beyotime Inst. Biotech, Beijing, China) according to the manufacturer's instructions. Protein concentrations were measured using a bicinchoninic acid protein assay kit (Beyotime Inst. Biotech, Beijing, China). A total of $30 \mu \mathrm{g}$ of protein was resolved by $10 \%$ SDS-polyacrylamide gel electrophoresis (SDS-PAGE) and transferred onto immunoblot polyvinylidene difluoride membranes (Millipore, Billerica, MA, USA). The blots were blocked with $5 \%$ nonfat milk in Tris-buffered saline with $0.1 \%$ Tween (TBS-T) for $1 \mathrm{~h}$, washed three times with TBS-T, and incubated overnight at $4^{\circ} \mathrm{C}$ with primary antibodies against iNOS (1:2000),
COX-2 (1:1000), OX-42 (1:1000), TH (1:1000) (Abcam, Cambridge, CA, USA), p-NF-кB p65 (1:1000) (Cell Signaling Technology, Danvers, MA, USA), GPR109A (1:300) and $\beta$-actin (1:2000) (Santa Cruz, CA, USA). The blots were then washed four times for $15 \mathrm{~min}$ each in TBS-T and incubated with a horseradish peroxidase-labeled secondary goat anti-rabbit (1:2000; Santa Cruz, CA, USA) or rabbit anti-goat antibody (1:2000; Santa Cruz, CA, USA) for $1 \mathrm{~h}$ at room temperature. Next, the blots were washed again four times for $15 \mathrm{~min}$ each in TBS-T. Membranes were visualized with enhanced chemiluminescence (ECL kit; Applygen Inst. Biotech, Beijing, China).

\section{Statistical analyses}

The data are presented as the mean \pm SD and were analyzed using SPSS 12.0 statistical software package (SPSS Inc., Chicago, IL, USA). The groups were compared by one-way analysis of variance (ANOVA) followed by the least significant difference test. A $P$ value of less than 0.05 was considered statistically significant.

\section{Results}

Effect of $\beta$-hydroxybutyric acid on lipopolysaccharideinduced degeneration of dopaminergic neurons

To investigate whether the LPS-induced damage of dopaminergic neurons could be prevented by BHBA, rat mesencephalic neuron-glia cultures were pretreated for $30 \mathrm{~min}$ with vehicle or $\mathrm{BHBA}(0,0.5,1$, or $1.5 \mathrm{mM})$ before treatment with $10 \mathrm{ng} / \mathrm{ml}$ LPS. Seven days later, the degeneration of dopaminergic neurons was assessed by $\mathrm{TH}$ immunostaining and $\left[{ }^{3} \mathrm{H}\right] \mathrm{DA}$ uptake. Morphologically, the remaining tyrosine hydroxylase-immunoreactive ( $\mathrm{TH}$-ir) neurons in the LPS-treated cultures had significantly fewer dendrites, and shorter or evenly truncated axons (Figure 1A). In the cultures pretreated with $1.5 \mathrm{mM}$ BHBA before LPS stimulation, the TH-ir neurons were more numerous and appeared less affected compared with the LPS-treated cultures (Figure 1A). The LPS treatment reduced the number of $\mathrm{TH}$-ir neurons by $75 \%(P<0.01)$ compared with the vehicletreated control cultures (Figure 1B). BHBA (1.5 mM) significantly attenuated the LPS-induced loss of TH-ir neurons (Figure 1B). $\left[{ }^{3} \mathrm{H}\right] \mathrm{DA}$ uptake assays showed that the LPS $(10 \mathrm{ng} / \mathrm{ml})$ treatment reduced the uptake capacity by approximately $70 \%$ of the vehicle control $(P<0.01)$, and this LPS-induced reduction in $\left[{ }^{3} \mathrm{H}\right] \mathrm{DA}$ was abated by pretreatment with BHBA in a concentrationdependent manner (Figure $1 \mathrm{C}$ ). $\left[{ }^{3} \mathrm{H}\right] \mathrm{DA}$ uptake in the cultures treated with $1.5 \mathrm{mM}$ BHBA alone did not differ from that in the control cultures (vehicle-treated only), suggesting that BHBA is devoid of obvious toxicity (Figure 1C). 
$\mathbf{A}$
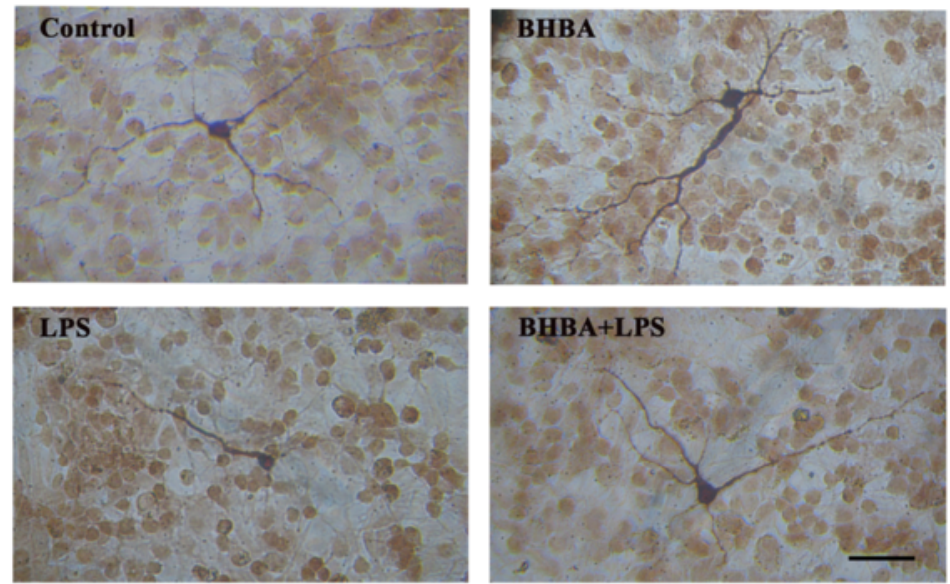

B

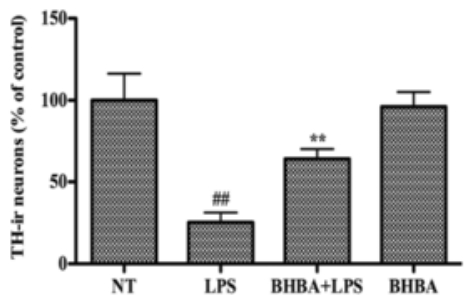

C

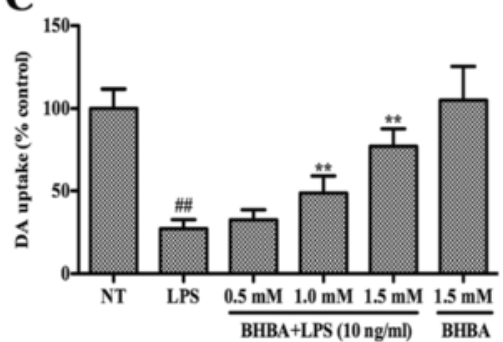

Figure 1 Effects of $\beta$-hydroxybutyric acid (BHBA) on lipopolysaccharide (LPS)-induced degeneration of dopaminergic neurons in mesencephalic neuron-glia cultures. Cultures were pretreated for 30 min with vehicle or indicated concentrations of BHBA before treatment with $10 \mathrm{ng} / \mathrm{ml}$ LPS. Seven days later, LPS-induced neurotoxicity was assessed by representative immunostaining images (A), the TH-ir neuron count (B), and the level of [ $\left.{ }^{3} \mathrm{H}\right] \mathrm{DA}$ uptake $(\mathbf{C})$. The scale bar indicates $250 \mu \mathrm{m}$. The results are expressed as a percentage of the vehicle-treated control cultures and presented as the mean \pm SD from three independent experiments performed in triplicate. ${ }^{* *} P<0.01$ compared with the

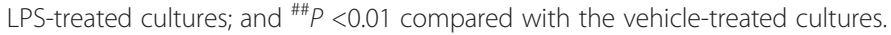

$\beta$-hydroxybutyric acid administration improves functional recovery from lipopolysaccharide intranigral injection

A rotational behavior assay in animal models of PD can be used to characterize the extent of a lesion and/or to investigate the therapeutic effects of drug candidates. To determine the effect of BHBA treatment on motor dysfunction, LPS-induced PD model rats were subjected to behavioral tests at two and four weeks after LPS injection. Administration of amphetamine, which is an indirect agonist of DA receptor, elicits rotational behavior towards the injection side. The results of the rotational behavior assay showed that the BHBA treatment significantly attenuated amphetamineinduced rotation (Figure 2). These data indicated that the administration of BHBA had beneficial effects on motor dysfunction in the LPS-induced PD model rats.

\section{$\beta$-hydroxybutyric acid administration attenuates depletion of dopamine and 3,4-dihydroxyphenylacetic acid in the striatum induced by lipopolysaccharide intranigral injection}

The levels of DA and its metabolite DOPAC, in the rat brain striatum were measured by HPLC. As shown in
Figure 3, in the vehicle-treated control group, the levels of DA and DOPAC on the LPS-injected side were reduced to $34 \%(P<0.01)$ and $41 \%(P<0.01)$ of the levels of the non-injected side, respectively. Treatment with BHBA $(0.4,0.8$, or $1.6 \mathrm{mmol} / \mathrm{kg} / \mathrm{d})$ for 4 weeks significantly attenuated DA depletion in the striatum as induced by LPS intranigral injection (Figure 3A). The levels of DA on the LPS-injected side were $49 \%(P<0.05), 72 \%(P<0.01)$ and 90\% $(P<0.01)$ of the levels of the noninjected side in the animals treated with $0.4,0.8$, and $1.6 \mathrm{mmol} / \mathrm{kg} / \mathrm{d}$ BHBA, respectively (Figure 3A). The levels of DOPAC on the LPS-injected side were $72 \%(P<0.01), 95 \%(P<0.01)$ and 91\% $(P<0.01)$ of the levels on the non-injected side in the groups treated with $0.4,0.8$, and $1.6 \mathrm{mmol} / \mathrm{kg} / \mathrm{d}$ BHBA, respectively (Figure $3 \mathrm{~B}$ ).

$\beta$-hydroxybutyric acid treatment increases the number of tyrosine hydroxylase (TH)-positive cells and TH expression in the substantia nigra of lipopolysaccharide-induced Parkinson's disease model rats

To further investigate the protective effect of BHBA on dopaminergic neurons, immunohistological analysis of 
A

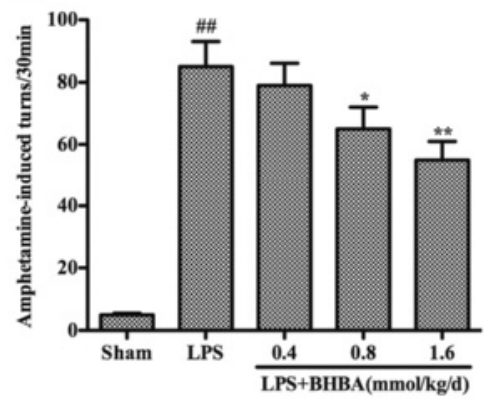

B

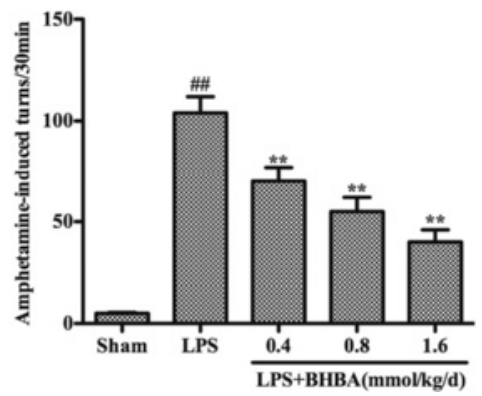

Figure $2 \beta$-hydroxybutyric acid (BHBA) treatment improves the behavioral dysfunction of lipopolysaccharide (LPS)-induced Parkinson's disease (PD) model rats. Rats were randomly grouped and then pretreated with BHBA $(0.4,0.8$, or $1.6 \mathrm{mmol} / \mathrm{kg} / \mathrm{d})$ or vehicle 3 days before LPS injection and subsequently for 21 days after LPS injection (24 days in total). (A, B) The number of turns induced by apomorphine for the LPS-induced PD model rats after 2 and 4 weeks of BHBA administration. The results are expressed as the mean \pm SD. ${ }^{*} P<0.05$ and ${ }^{* *} P<0.01$ compared with the LPS-treated rats; and ${ }^{\#} P<0.01$ compared with the sham-operated control rats.

$\mathrm{TH}$ expression was carried out in an in vivo study. In sham-operated animals, the numbers of TH-ir neurons were similar on the ipsilateral and contralateral sides to the injection site (Figure 4A). The survival rate of the TH-ir neurons was 95\% (Figure 4F). The animals that received the vehicle treatment after LPS intranigral injection showed marked losses of TH-ir neurons and their dendrites (Figure 4B). Only 19\% of the TH-ir neurons $(P<0.01)$ in the SNpc on the LPS-injected side survived compared with those on the noninjected side (Figure 4F). In contrast, treatment with $0.4,0.8$, or $1.6 \mathrm{mmol} / \mathrm{kg} / \mathrm{d}$ BHBA dramatically rescued this decline (Figure $4 \mathrm{C}-4 \mathrm{~F}$ ). $\mathrm{TH}$, which is the rate-limiting enzyme in the synthesis of catecholamines, is critically involved in DA synthesis. We further investigated the expression of $\mathrm{TH}$ in the $\mathrm{SN}$ using western blot analysis. The results showed that the expression of $\mathrm{TH}$ significantly decreased in the SN of the LPS-induced PD model rats. BHBA administration markedly increased $\mathrm{TH}$ expression (Figure 4G), indicating that it rescued dopaminergic neuronal damage caused by LPS-induced neurotoxicity.

\section{$\beta$-hydroxybutyric acid treatment inhibits microglial activation induced by lipopolysaccharide intranigral injection}

To investigate whether the neuroprotective effect of BHBA is associated with the inhibition of LPS-induced microglial activation, we examined the expression of IBA-1, which is a specific marker for microglial activation. The activation of microglia was significantly suppressed by BHBA treatment in a dose-dependent manner (Figure 5A). To obtain quantitative data, the $\mathrm{SN}$ of the rats was dissected out, and microglial activation was determined by western blot analysis using an OX-42 antibody. The results confirmed that the BHBA treatment suppressed LPS-induced microglial activation (Figure 5B).

Because microglia are the main sources of proinflammatory enzymes and pro-inflammatory cytokines in the brain and BHBA inhibits microglial activation, we hypothesized that BHBA treatment could inhibit the LPS-induced expression of pro-inflammatory enzymes and pro-inflammatory cytokines. We measured the mRNA
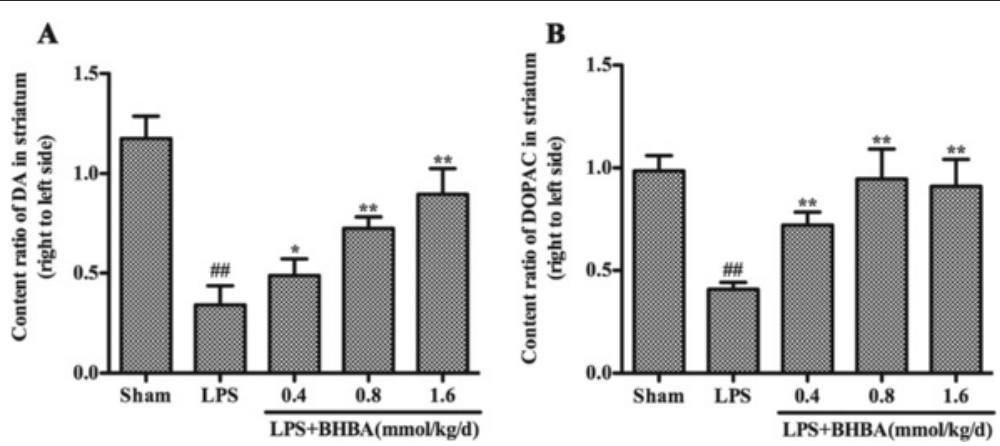

Figure 3 Effects of $\beta$-hydroxybutyric acid (BHBA) treatment on the levels of dopamine (DA) and 3,4-dihydroxyphenylacetic acid (DOPAC) in the striatum. Rats were randomly grouped and then pretreated with BHBA $(0.4,0.8$, or $1.6 \mathrm{mmol} / \mathrm{kg} / \mathrm{d})$ or vehicle 3 days before lipopolysaccharide (LPS) injection and subsequently for 21 days after LPS injection (24 days in total). The levels of DA (A) and DOPAC (B) in the striatum were detected by high-performance liquid chromatography (HPLC), and the ratio of the right to left side was calculated. The results are expressed as the mean \pm SD. ${ }^{*} P<0.05$ and ${ }^{* *} P<0.01$ compared with the LPS-treated rats; and ${ }^{\# \#} P<0.01$ compared with the sham-operated control rats. 

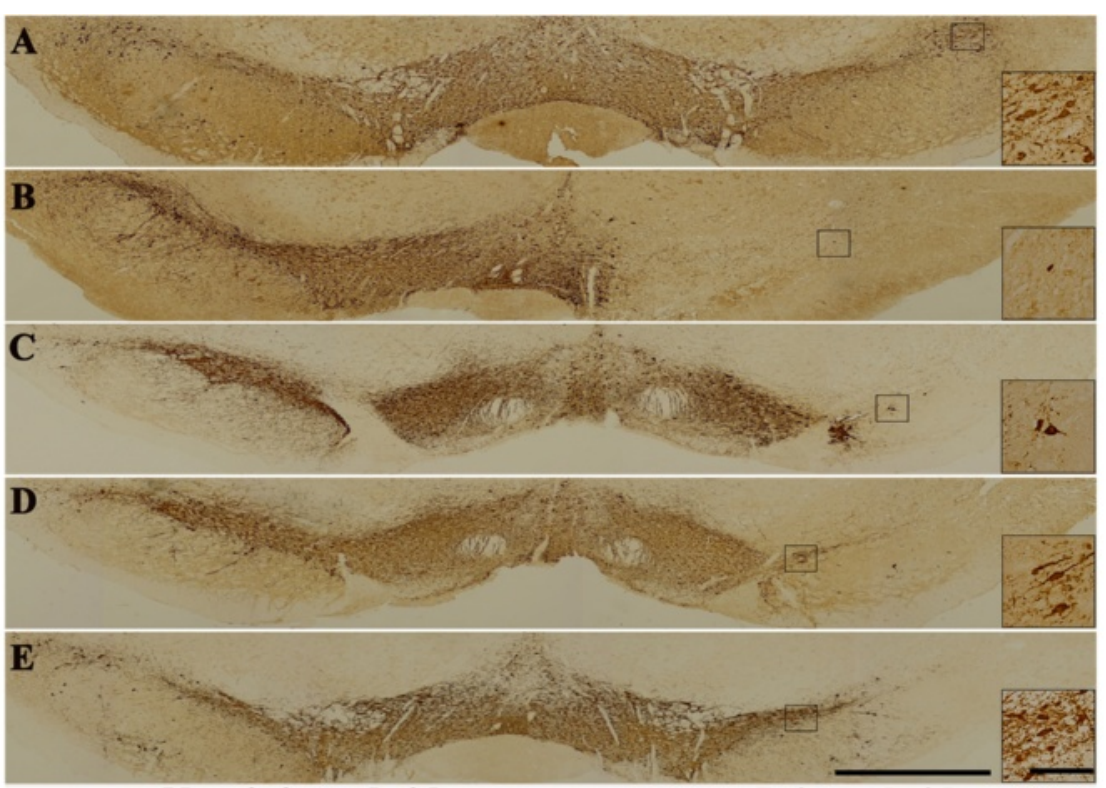

Non-injected side

Injected side

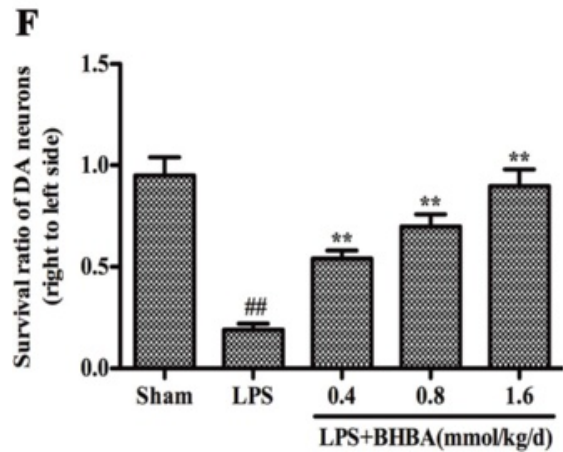

$\mathbf{G}$

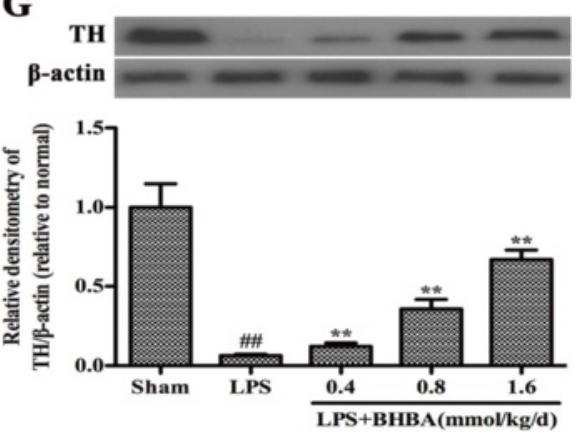

Figure $4 \beta$-hydroxybutyric acid (BHBA) treatment increases the number of tyrosine hydroxylase (TH)-positive cells and TH expression in the substantia nigra (SN) of lipopolysaccharide (LPS)-induced Parkinson's disease (PD) model rats. PBS or $10 \mu \mathrm{g}$ LPS was unilaterally injected into the right SN of rats. The animals were sacrificed after 4 weeks of BHBA treatment. (A-E) Staining of TH-positive neurons in the SN. SN brain sections were processed for TH immunostaining. Insets are higher magnifications taken from the area outlined in lower magnification pictures. Scale bar of inset, $100 \mu \mathrm{m}$; scale bar of low magnification images, $1.0 \mathrm{~mm}$. (F) The survival ratio of the dopaminergic neurons in the SNpc (the injected side versus the noninjected side) was calculated. (G) Western blot assay of TH expression. The experiments were repeated three times. A representative immunoblot is shown. The results are expressed as the mean \pm SD. ${ }^{*} P<0.05$ and ${ }^{* *} P<0.01$ compared with the LPS-treated

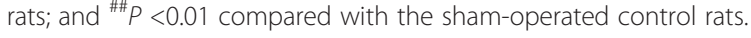

expression of iNOS, COX-2, TNF- $\alpha$, IL-1 $\beta$, and IL-6. As shown in Figure $5 \mathrm{C}-\mathrm{G}$, LPS injection significantly upregulated iNOS, COX-2, TNF- $\alpha$, IL-1 $\beta$, and IL-6 mRNA expression, and the BHBA treatment downregulated those expressions in a dose-dependent manner (Figure 5C-G).

\section{Lipopolysaccharide enhances expression of GPR109A in primary rat microglial cells}

GPR109A is the functional receptor of BHBA, and its mRNA (Figure 6A) and protein (Figure 6B) were detected in primary rat microglial cells. To investigate whether a correlation exists between GPR109A expression and the degree of microglial activation, microglia were stimulated with LPS
(0, 0.5, 1 , or $10 \mathrm{ng} / \mathrm{ml})$ for several time points. GPR109A mRNA expression was detected as early as $4 \mathrm{~h}$ after LPS stimulation and was observed to significantly increase in both dose- and time-dependent manners (Figure 6C). This finding revealed that GPR109A mRNA is expressed at low levels in unstimulated conditions and is induced in a timedependent fashion in response to LPS, suggesting a role of GPR109A during the early stages of microglial activation.

B-hydroxybutyric acid inhibits lipopolysaccharide-induced inflammation responses via GPR109A in primary rat microglial cells

The incubations of non-siRNA-transfected, scrambled siRNA-transfected and GPR109A-siRNA transfected primary 


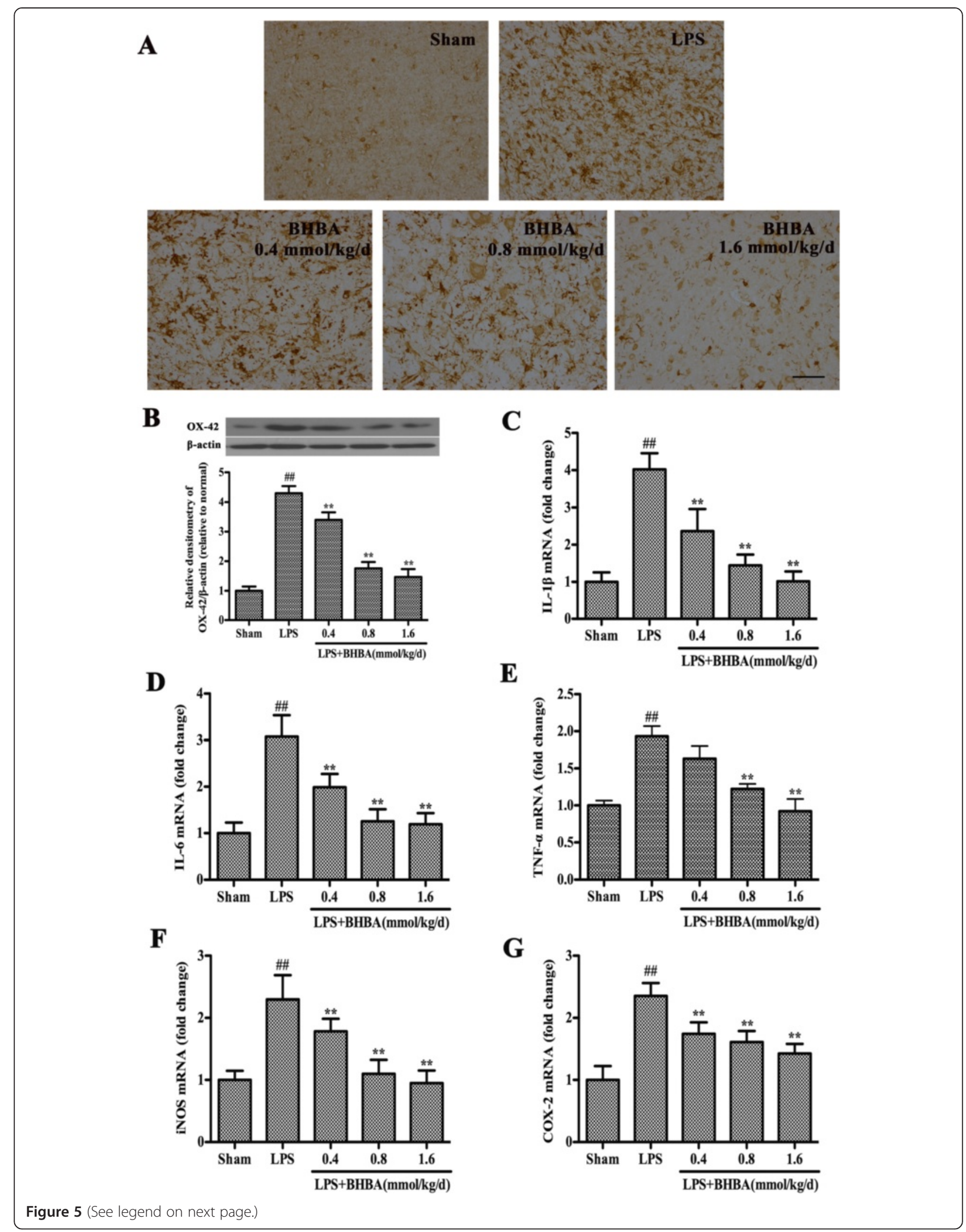


(See figure on previous page.)

Figure $5 \beta$-hydroxybutyric acid (BHBA) treatment inhibits microglial activation and downregulates mRNA expression of pro-inflammatory mediators in the substantia nigra (SN) of lipopolysaccharide (LPS)-induced Parkinson's disease (PD) model rats. (A) The morphological changes of the microglia in the SN as shown by IBA-1 immunostaining. Representative photomicrographs of the SN area are shown. The scale bar indicates $100 \mu \mathrm{m}$. (B) Western blot assay of O-X42 expression. The experiments were repeated three times. A representative immunoblot is shown. (C-G) Real-time RT-PCR analysis of pro-inflammatory enzyme (iNOS and COX-2) and pro-inflammatory cytokine (TNF-a, IL-1 1 , and IL-6) expression in the SN of LPS-induced PD model rats. The data are expressed as fold changes relative to the sham-operated control rats. The results are expressed as the mean \pm SD. ${ }^{*} P<0.05$ and ${ }^{* *} P<0.01$ compared with the LPS-treated rats; and ${ }^{\# \#} P<0.01$ compared with the sham-operated control rats.

rat microglial cells were carried out in parallel. As shown in Figures 7 and 8, pretreatment with BHBA (1.5 mM) attenuated the LPS-induced increased production of iNOS, COX-2, TNF- $\alpha$, IL- $1 \beta$ and IL- 6 in both the nontransfected and scrambled siRNA-transfected cells, but in the cells with the knockdown of GPR109A by siRNA, this effect was abolished. These data suggest that BHBA inhibits the LPS-induced production of pro-inflammatory enzyme (iNOS and COX-2) and pro-inflammatory cytokine (TNF- $\alpha$, IL-1 $\beta$, and IL-6) through GPR109A.

\section{$\beta$-hydroxybutyric acid downregulates NF-KB activation via GPR109A}

The NF-kB pathway is a key mediator of inflammation and is activated via toll-like receptors (TLRs), resulting in increased cytokine and chemokine production [32]. Moreover, transcription of iNOS, COX-2, TNF- $\alpha$, IL- $1 \beta$, and IL- 6 is regulated by the transcription factor NF- $\mathrm{kB}$. To elucidate the inhibitory mechanism of BHBA on pro-inflammatory mediator production in primary rat microglial cells, we examined NF- $\mathrm{kB}$ signaling in response to LPS in primary rat microglial cells. Microglial cells were stimulated with LPS $(10 \mathrm{ng} / \mathrm{ml})$ for $0.25,0.5,1,2,4$ and $6 \mathrm{~h}$ in the presence or absence of BHBA $(1.5 \mathrm{mM})$. Cell lysates were subjected to western blotting for $\mathrm{p}-\mathrm{NF}-\mathrm{k} B$ p65, NF-kB p65 and $\beta$-actin. As shown in Figure 9, the level of active NF- $\mathrm{kB}$ p65 (p-NF- $\mathrm{kB}$ p65) peaked at $0.25 \mathrm{~h}$ after LPS stimulation. Levels of $\mathrm{p}-\mathrm{NF}-\mathrm{kB}$ p65 were maintained for $4 \mathrm{~h}$; however, a striking reduction in its level was observed at $2 \mathrm{~h}$ after LPS stimulation (Figure 9A,B). As expected, BHBA significantly reduced its levels in primary rat microglial cells after LPS stimulation (Figure 9A,B). Knockdown of GPR109A with siRNA abolished this effect (Figure 9C,D).

\section{Discussion}

Our findings demonstrated that BHBA exerted neuroprotective effects on dopaminergic neurons by inhibiting microglial activation in an in vitro model of LPS-induced dopaminergic neurodegeneration and an in vivo rat model induced by intranigral injection of LPS. The mechanistic study showed that the inhibitory effect of BHBA on microglia was mediated by GPR109A and involved the NF- $\mathrm{kB}$ signaling pathway, inhibiting pro-inflammatory enzyme (iNOS and COX-2) and pro-inflammatory cytokine
(TNF- $\alpha$, IL-1 $\beta$, and IL-6) production. These data revealed that GPR109A-mediated signaling pathways might represent potential targets for therapeutic interventions to prevent or slow the progression of PD.

In recent years, the involvement of neuroinflammatory processes in the nigral degeneration of dopaminergic neurons in $\mathrm{PD}$ has gained increasing attention. In the CNS, microglia, which are the resident innate immune cells, play a major role in the inflammatory process. In addition, these cells have been found to be highly concentrated in the SNpc $[18,33]$. They are the resident macrophages of the brain and share similar properties [34,35] constituting $10 \%$ of brain cells [33]. Once activated, these microglia transform from striated bodies into large round, amoeboid, bodies with short, thick processes. In PD, activated microglia in the SNpc have been found to express pro-inflammatory enzyme (iNOS and COX-2) and pro-inflammatory cytokine (TNF- $\alpha$, IL-1 $\beta$, and IL-6) $[17,36]$. Most evidence has indicated that pro-inflammatory enzymes and pro-inflammatory cytokines may mediate neuronal degeneration [37-39].

LPS, which is an endotoxin from Gram-negative bacteria, is a potent stimulator of microglia, and in vivo and in vitro PD models induced by LPS are widely used to study the inflammatory process in the pathogenesis of PD. These PD models have also been widely used in drug discovery, and a variety of agents have been evaluated for their potential neuroprotective effects in LPS-induced PD models, such as FLZ, triptolide, and urocortin [21,22,40]. In a mesencephalic mixed neuron-glial culture, LPS have been shown to induce microglial activation, and activated microglia have been demonstrated to release the proinflammatory and cytotoxic factors NO, TNF- $\alpha$, and IL- $1 \beta$, leading to the consequent degeneration of dopaminergic neurons [20]. LPS injected into the $\mathrm{SN}$ of rats induce microglial activation and dopaminergic neuron loss [41]. Moreover, there is no detectable damage to either GABAergic or serotoninergic neurons in the striatum and nigra after LPS injection, indicating that LPS selectively induce dopaminergic neuron death in the nigrostriatal system [42]. More recent studies have confirmed these results, also finding increased levels of proinflammatory mediators, including IL- $1 \beta$, TNF- $\alpha$, IL- 6 , and NO, in the SN after LPS injection, which may be causal factor of LPS-induced neuronal damage $[21,43,44]$. In addition, the 

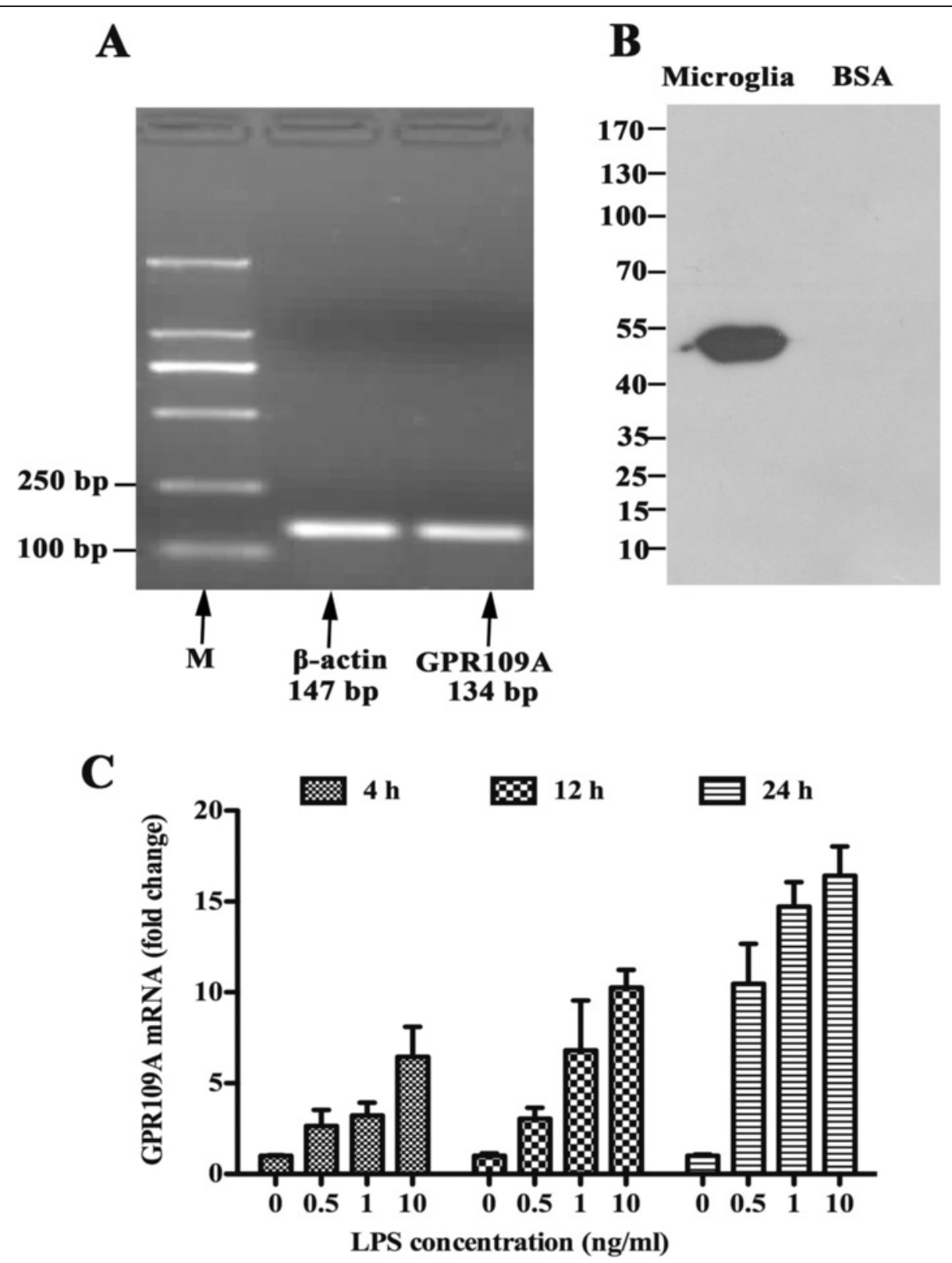

Figure 6 Lipopolysaccharide (LPS) enhances expression of GPR109A in primary rat microglial cells. (A) RT mixtures from primary rat microglial cells were carried out to detect GPR109A mRNA expression by PCR amplification (M, 2000 bp DNA marker). PCR products were visualized by $2 \%$ agarose gel electrophoresis, and the expected 134-bp GPR109A was detected in the primary rat microglial cells. (B) Western blot of GPR109A in primary rat microglial cells showing a specific band of the expected size at approximately $50 \mathrm{kDa}$. (C) Microglial cells were treated with $0,0.5,1$, or $10 \mathrm{ng} / \mathrm{ml}$ of LPS for the indicated times. GPR109A mRNA expression was quantified by quantitative real-time RT-PCR and normalized to $\beta$-actin mRNA expression.

effects of intranigral LPS injection on behavior and DA content and turnover have been investigated, and it has been shown that LPS treatment enhances locomotor activity two- to threefold and increases DA turnover ratios in comparison with control subjects. These findings suggest that LPS insult may induce a compensatory response of the dopaminergic system [22]. Therefore, in vitro and in vivo LPS PD models represent powerful tools for mechanistic studies and the identification of potential therapeutic agents.

BHBA is an important intermediate of amino and fatty acid catabolism that has been reported to be effective in the treatment of a variety of inflammatory and autoimmune diseases, such as colonic inflammation and experimental allergic encephalomyelitis (EAE) [45,46]. A previous study has reported that BHBA has potent neuroprotective effects 


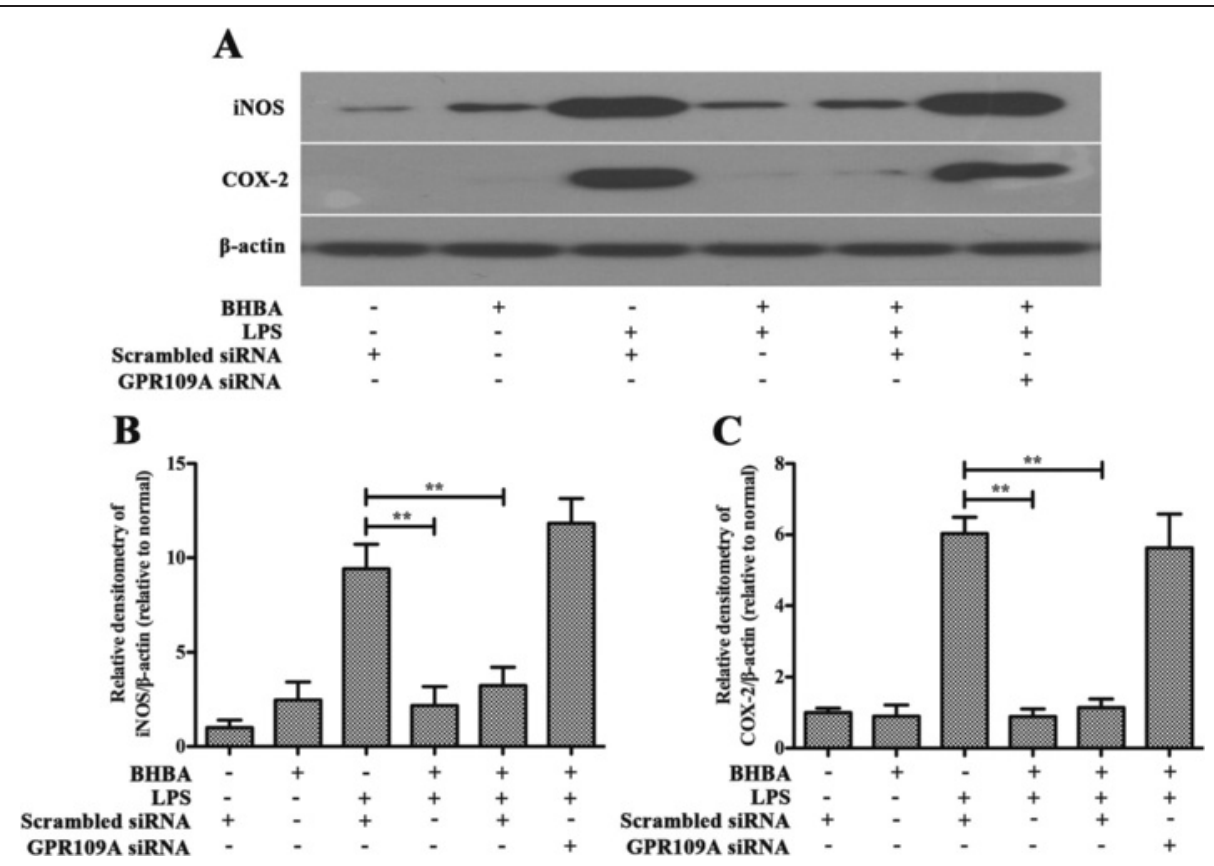

Figure $7 \beta$-hydroxybutyric acid (BHBA) inhibits lipopolysaccharide (LPS)-Induced production of pro-inflammatory enzymes via GPR109A in primary rat microglial cells. Attenuation by BHBA $(1.5 \mathrm{mM})$ of LPS-induced production of iNOS (A, B) and COX-2 (A, C) from primary rat microglial cells in vitro, this effect is abolished with silencing of GPR109A (**P<0.01).

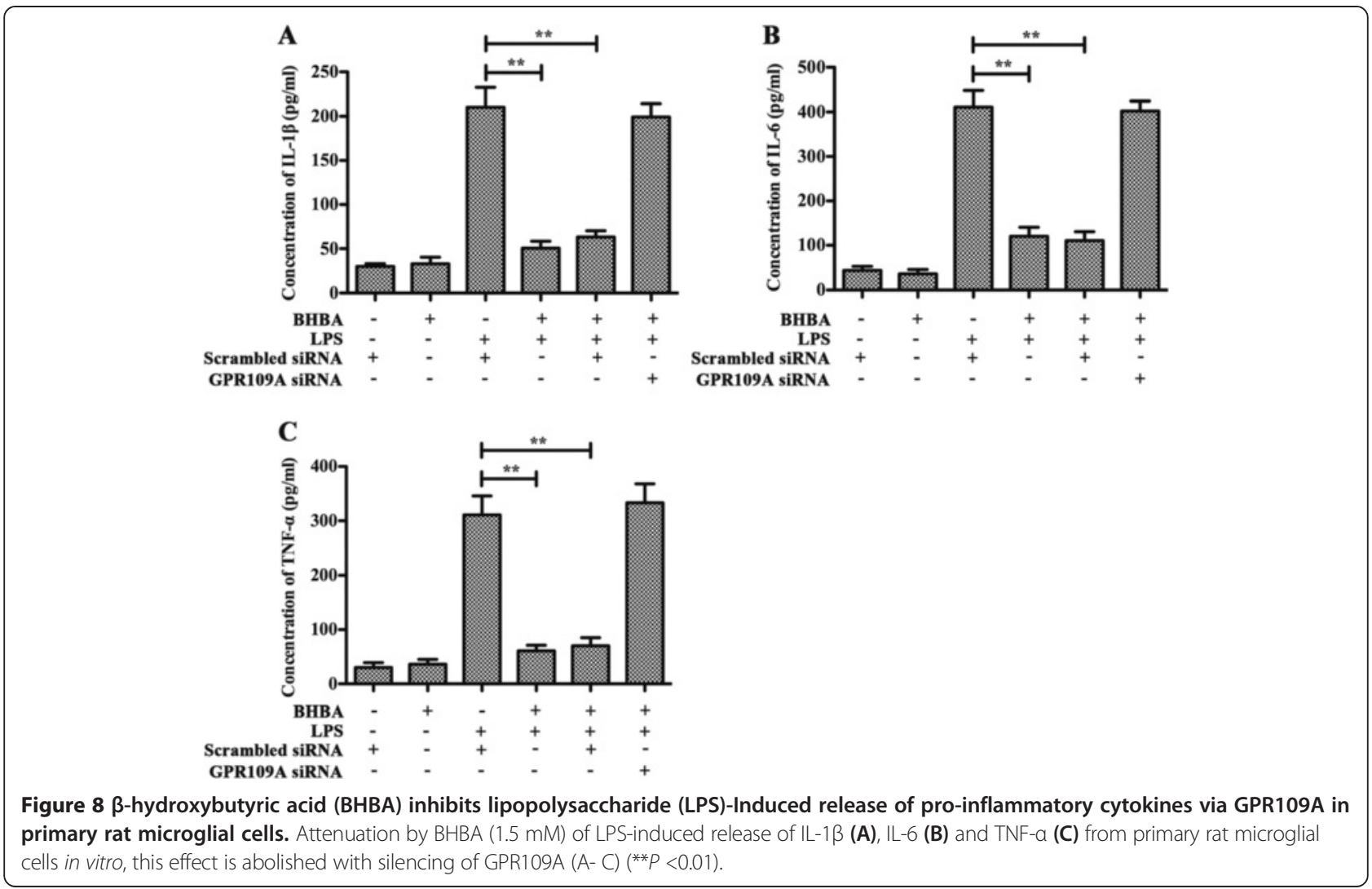




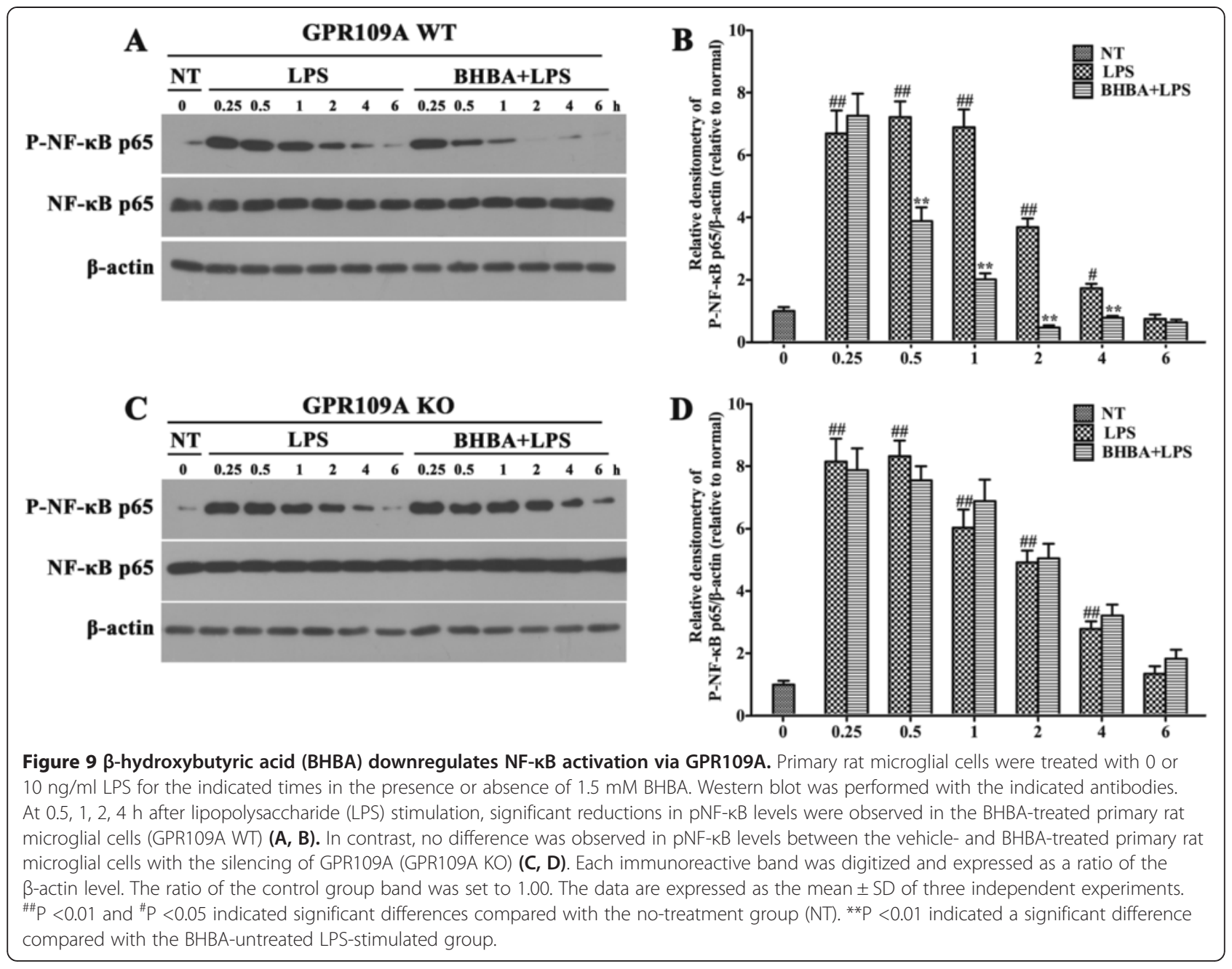

on dopaminergic neurons both in vitro and in vivo. Yoshihiro et al. have found that BHBA protects cultured mesencephalic neurons from $\mathrm{MPP}^{+}$toxicity and hippocampal neurons from $A \beta_{1-42}$ toxicity [24]. In vivo administration of BHBA confers partial protection against dopaminergic neurodegeneration and motor deficits induced by MPTP, and these effects appear to be mediated by a complex II-dependent mechanism that leads to improved mitochondrial respiration and ATP production [25]. Soyeon et al. have proven that BHBA extends the life span, attenuates motor deficits, and prevents striatal histone deacetylation in transgenic R6/2 mice [47]. To elucidate whether its neuroprotective activity involves an anti-inflammatory function, we investigated the effect of BHBA on LPS-induced damage to dopaminergic neurons in a primary mesencephalic neuron/glia mixed culture. We found that BHBA concentration-dependently attenuated the LPS-induced decrease in $\left[{ }^{3} \mathrm{H}\right] \mathrm{DA}$ uptake and loss of TH-ir neurons in a primary mesencephalic neuron/glia mixed culture. In the current in vivo study, we investigated the motor dysfunction of these PD model rats using a rotational behavior assay. Because LPS was injected on one side of the $\mathrm{SN}$, apomorphine-induced rotation to the lesioned side was used to evaluate the degree of damage to the dopaminergic system. Apomorphine-induced rotation significantly increased in the LPS-induced PD model rats, and BHBA showed therapeutic effects on this behavioral dysfunction. Further experiments demonstrated that BHBA inhibited LPS-induced microglial overactivation, pro-inflammatory factor release and dopaminergic neuronal damage. These data suggest that BHBA plays a neuroprotective role through inhibiting microglial overactivation.

GPR109A (PUMA-G in mice and HM74A in humans) is a seven-transmembrane G-protein-coupled receptor of the Gi family that is expressed mainly in white adipocytes and immune cells, such as monocytes and neutrophils [47]. BHBA has been identified as an endogenous ligand of GPR109A [48]. The anti-inflammatory effects of BHBA are mediated by the activation of GPR109A [47]. Accumulating data have demonstrated a strong anti-inflammatory activity 
of BHBA in macrophages, monocytes, adipocytes, and retinal pigment epithelial cells. In vitro experiments have demonstrated that BHBA inhibits pro-inflammatory cytokine production, LDL uptake, and chemotaxis in macrophages via activating GPR109A [49]. Moreover, BHBA inhibits the expression of TNF- $\alpha$, IL- 6 and MCP-1 in human monocytes stimulated by LPS [50]. In vivo experiments have shown that GPR109A mediates the therapeutic effects of DMF in EAE [46]. In this study, we found that the level of GPR109A expression was correlated with the degree of microglial activation, as measured by proinflammatory cytokine production. Therefore, we hypothesized that activated microglia may be subjected to negative feedback mechanisms via GPR109A signaling.

We further assessed the mechanism underlying the anti-inflammatory effect of BHBA in primary rat microglial cells and found that it significantly inhibited LPS-induced proinflammatory mediator production. The knockdown of GPR109A with siRNA resulted in the loss of this anti-inflammatory effect in primary rat microglial cells. Because NF- $\mathrm{KB}$ is clearly one of the most important regulators of pro-inflammatory gene expression [51], we examined whether GPR109A-mediated signaling pathways modulate NF- $\mathrm{kB}$ signaling and found that BHBA inhibits pro-inflammatory cytokines via NF- $\mathrm{KB}$ inactivation in primary rat microglial cells. Furthermore, we demonstrated that the inhibitory effect of BHBA is mediated by GPR109A.

\section{Conclusions}

In conclusion, this study demonstrates that BHBA treatment improves LPS-induced behavioral dysfunction and protects dopaminergic neurons through inhibiting microgliamediated neuroinflammation both in vitro and in vivo. Several lines of evidence presented in this study demonstrate that BHBA provides potent neuroprotection to dopaminergic neurons against LPS-induced neurotoxicity through the regulation of GPR109A-mediated signaling pathways. Thus, GPR109A-mediated signaling pathways may represent potential targets for therapeutic intervention to prevent or slow the progression of PD.

\footnotetext{
Abbreviations

BHBA: $\beta$-hydroxybutyric acid; COX-2: Cyclooxygenase-2; DA: Dopamine; DMEM: Dulbecco's modified Eagle medium; FBS: Fetal bovine serum; GPR109A: G-protein-coupled receptor 109A; IBA-1: Ionized calcium binding adaptor molecule-1; IL-6: Interleukin 6; iNOS: Inducible nitric oxide synthase;

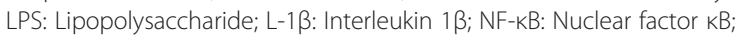
PBS: Phosphate-buffered saline; PD: Parkinson's disease; SN: Substantia nigra; TH: Tyrosine hydroxylase; TNF-a: Tumor necrosis factor alpha.
}

\section{Competing interests}

The authors declare that they have no competing interests.

\section{Authors' contributions}

SPF, JFW, WJX, HML, BRL, YLZ, SNL, BXH and QKL performed the experiments; WW and JXL designed the study; and SPF wrote the manuscript. All authors read and approved the final manuscript.

\section{Acknowledgements}

This work was founded by National Key Basic Research Program of China (project no. 2011CB100805), National Nature Science Foundation of China (project no. 31372396), Graduate Innovation Fund of Jilin University (project no. 2014117), Jilin Scientific and Technological Development Program (project no. 20130206036NY), Veterinary Medicine Key Lab of Heilongjiang Province (No. AMKL2012003).

Received: 11 October 2014 Accepted: 21 December 2014

Published online: 17 January 2015

\section{References}

1. Dorsey ER, Constantinescu R, Thompson JP, Biglan KM, Holloway RG, Kieburtz K, et al. Projected number of people with Parkinson disease in the most populous nations, 2005 through 2030. Neurology. 2007;68:384-6.

2. Braak H, Del Tredici K, Rub U, de Vos RAI, Steur ENHJ, Braak E. Staging of brain pathology related to sporadic Parkinson's disease. Neurobiol Aging. 2003;24:197-211.

3. Blum D, Torch S, Lambeng N, Nissou MF, Benabid AL, Sadoul R, et al. Molecular pathways involved in the neurotoxicity of 6-OHDA, dopamine and MPTP: contribution to the apoptotic theory in Parkinson's disease. Prog Neurobiol. 2001;65:135-72.

4. Collier TJ, Sortwell CE. Therapeutic potential of nerve growth factors in Parkinson's disease. Drugs Aging. 1999;14:261-87.

5. Jankovic J. Parkinson's disease: clinical features and diagnosis. J Neurol Neurosurg Psychiatry. 2008;79:368-76.

6. Jenner P. Oxidative stress and Parkinson's disease. Handb Clin Neurol. 2007;83:507-20.

7. Perry VH. Innate inflammation in Parkinson's disease. Cold Spring Harb Perspect Med. 2012;2:a009373.

8. Keane PC, Kurzawa M, Blain PG, Morris CM. Mitochondrial dysfunction in Parkinson's disease. Parkinsons Dis. 2011;2011:716871.

9. Gyoneva S, Shapiro L, Lazo C, Garnier-Amblard E, Smith Y, Miller GW, et al. Adenosine A2A receptor antagonism reverses inflammation-induced impairment of microglial process extension in a model of Parkinson's disease. Neurobiol Dis. 2014;67:191-202.

10. Deleidi M, Gasser T. The role of inflammation in sporadic and familial Parkinson's disease. Cell Mol Life Sci. 2013;70:4259-73.

11. Hirsch EC, Hunot S, Damier P, Faucheux B. Glial cells and inflammation in Parkinson's disease: a role in neurodegeneration? Ann Neurol. 1998;44:S115-20.

12. Kitamura Y, Itano Y, Kubo T, Nomura Y. Suppressive effect of FK-506, a novel immunosuppressant, against MPTP-induced dopamine depletion in the striatum of young C57BL/6 mice. J Neuroimmunol. 1994;50:221-4.

13. Kurkowska-Jastrzebska I, Wronska A, Kohutnicka M, Czlonkowski A, Czlonkowska A. The inflammatory reaction following 1-methyl-4-phenyl-1,2,3, 6-tetrahydropyridine intoxication in mouse. Exp Neurol. 1999;156:50-61.

14. Wang MJ, Huang HY, Chen WF, Chang HF, Kuo JS. Glycogen synthase kinase-3beta inactivation inhibits tumor necrosis factor-alpha production in microglia by modulating nuclear factor kappaB and MLK3/JNK signaling cascades. J Neuroinflammation. 2010;7:99.

15. Fu SP, Li SN, Wang JF, Li Y, Xie SS, Xue WJ, et al. BHBA suppresses LPS-induced inflammation in BV-2 cells by inhibiting NF-KB activation. Mediators Inflamm. 2014;2014:983401.

16. Liu YX, Qin LY, Li GR, Zhang W, An LJ, Liu B, et al. Dextromethorphan protects dopaminergic neurons against inflammation-mediated degeneration through inhibition of microglial activation. J Pharmacol Exp Ther. 2003;305:212-8.

17. Li FQ, Lu XZ, Liang XB, Zhou HF, Xue B, Liu XY, et al. Triptolide, a Chinese herbal extract, protects dopaminergic neurons from inflammation-mediated damage through inhibition of microglial activation. J Neuroimmunol. 2004;148:24-31.

18. Kim WG, Mohney RP, Wilson B, Jeohn GH, Liu B, Hong JS. Regional difference in susceptibility to lipopolysaccharide-induced neurotoxicity in the rat brain: Role of microglia. J Neurosci. 2000;20:6309-16.

19. Liu B, Gao HM, Wang JY, Jeohn GH, Cooper CL, Hong JS. Role of nitric oxide in inflammation-mediated neurodegeneration. Nitric Oxide. 2002;962:318-31.

20. Gayle DA, Ling Z, Tong C, Landers T, Lipton JW, Carvey PM. Lipopolysaccharide (LPS)-induced dopamine cell loss in culture: roles of tumor necrosis factor-alpha, interleukin-1beta, and nitric oxide. Brain Res Dev Brain Res. 2002;133:27-35. 
21. Zhou HF, Liu XY, Niu DB, Li FQ, He QH, Wang XM. Triptolide protects dopaminergic neurons from inflammation-mediated damage induced by lipopolysaccharide intranigral injection. Neurobiol Dis. 2005;18:441-9.

22. Tai W, Ye X, Bao X, Zhao B, Wang X, Zhang D. Inhibition of Src tyrosine kinase activity by squamosamide derivative FLZ attenuates neuroinflammation in both in vivo and in vitro Parkinson's disease models. Neuropharmacology. 2013;75:201-12.

23. Izumi Y, Ishii K, Katsuki H, Benz AM, Zorumski CF. beta-Hydroxybutyrate fuels synaptic function during development. Histological and physiological evidence in rat hippocampal slices. J Clin Invest. 1998;101:1121-32.

24. Kashiwaya Y, Takeshima T, Mori N, Nakashima K, Clarke K, Veech RL. D-beta-hydroxybutyrate protects neurons in models of Alzheimer's and Parkinson's disease. Proc Natl Acad Sci U S A. 2000;97:5440-4.

25. Tieu K, Perier C, Caspersen C, Teismann P, Wu DC, Yan SD, et al D-beta-hydroxybutyrate rescues mitochondrial respiration and mitigates features of Parkinson disease. J Clin Invest. 2003;112:892-901.

26. Lim S, Chesser AS, Grima JC, Rappold PM, Blum D, Przedborski S, et al. D-beta-hydroxybutyrate is protective in mouse models of Huntington's disease. PLoS One. 2011:6:e24620.

27. Paxinos $G$, Watson $C$. The rat brain in stereotaxic coordinates. 6 th ed. Elsevier; 2006.

28. Liu B, Jiang JW, Wilson BC, Du L, Yang SN, Wang JY, et al. Systemic infusion of naloxone reduces degeneration of rat substantia nigral dopaminergic neurons induced by intranigral injection of lipopolysaccharide. J Pharmacol Exp Ther. 2000:295:125-32.

29. Qin L, Liu Y, Cooper C, Liu B, Wilson B, Hong JS. Microglia enhance beta-amyloid peptide-induced toxicity in cortical and mesencephalic neurons by producing reactive oxygen species. J Neurochem. 2002;83:973-83.

30. Gebicke-Haerter PJ, Bauer J, Schobert A, Northoff H. Lipopolysaccharide-free conditions in primary astrocyte cultures allow growth and isolation of microglial cells. J Neurosci. 1989;9:183-94.

31. Wang K, Yuan CP, Wang W, Yang ZQ, Cui W, Mu LZ, et al. Expression of interleukin 6 in brain and colon of rats with TNBS-induced colitis. World J Gastroenterol. 2010;16:2252-9.

32. Dai JN, Zong Y, Zhong LM, Li YM, Zhang W, Bian LG, et al. Gastrodin Inhibits Expression of Inducible NO Synthase, Cyclooxygenase-2 and Proinflammatory Cytokines in Cultured LPS-Stimulated Microglia via MAPK Pathways. Plos One. 2011;6:e21891.

33. Lawson LJ, Perry VH, Dri P, Gordon S. Heterogeneity in the distribution and morphology of microglia in the normal adult mouse brain. Neuroscience. 1990;39:151-70

34. Sedgwick JD, Schwender S, Imrich H, Dorries R, Butcher GW, ter Meulen V. Isolation and direct characterization of resident microglial cells from the normal and inflamed central nervous system. Proc Natl Acad Sci U S A. 1991;88:7438-42.

35. Perry VH, Bell MD, Brown HC, Matyszak MK. Inflammation in the nervous system. Curr Opin Neurobiol. 1995;5:636-41.

36. Vijitruth R, Liu M, Choi DY, Nguyen XV, Hunter RL, Bing G. Cyclooxygenase-2 mediates microglial activation and secondary dopaminergic cell death in the mouse MPTP model of Parkinson's disease. J Neuroinflammation. 2006:3:6.

37. Koprich JB, Reske-Nielsen C, Mithal P, Isacson O. Neuroinflammation mediated by IL-1 beta increases susceptibility of dopamine neurons to degeneration in an animal model of Parkinson's disease. J Neuroinflammation. 2008;5:8.

38. Li M, Dai FR, Du XP, Yang QD, Chen Y. Neuroprotection by silencing iNOS expression in a 6-OHDA model of Parkinson's disease. J Mol Neurosci. 2012:48:225-33

39. Reale M, larlori C, Thomas A, Gambi D, Perfetti B, Di Nicola M, et al. Peripheral cytokines profile in Parkinson's disease. Brain Behav Immun. 2009;23:55-63.

40. Abuirmeileh A, Lever R, Kingsbury AE, Lees AJ, Locke IC, Knight RA, et al. The corticotrophin-releasing factor-like peptide urocortin reverses key deficits in two rodent models of Parkinson's disease. Eur J Neurosci. 2007;26:417-23.

41. Hoban DB, Connaughton E, Connaughton C, Hogan G, Thornton C, Mulcahy $P$, et al. Further characterisation of the LPS model of Parkinson's disease: A comparison of intra-nigral and intra-striatal lipopolysaccharide administration on motor function, microgliosis and nigrostriatal neurodegeneration in the rat. Brain Behav Immun. 2013;27:91-100
42. Herrera AJ, Castano A, Venero JL, Cano J, Machado A. The single intranigral injection of LPS as a new model for studying the selective effects of inflammatory reactions on dopaminergic system. Neurobiol Dis. 2000;7:429-47

43. Hernandez-Romero MC, Arguelles S, Villaran RF, de Pablos RM, Delgado-Cortes MJ, Santiago M, et al. Simvastatin prevents the inflammatory process and the dopaminergic degeneration induced by the intranigral injection of lipopolysaccharide. J Neurochem. 2008;105:445-59.

44. Lu X, Bing G, Hagg T. Naloxone prevents microglia-induced degeneration of dopaminergic substantia nigra neurons in adult rats. Neuroscience. 2000:97:285-91.

45. Singh N, Gurav A, Sivaprakasam S, Brady E, Padia R, Shi H, et al. Activation of Gpr109a, receptor for niacin and the commensal metabolite butyrate, suppresses colonic inflammation and carcinogenesis. Immunity. 2014:40:128-39.

46. Chen H, Assmann JC, Krenz A, Rahman M, Grimm M, Karsten CM, et al. Hydroxycarboxylic acid receptor 2 mediates dimethyl fumarate's protective effect in EAE. J Clin Invest. 2014;124:2188-92.

47. Offermanns S. The nicotinic acid receptor GPR109A (HM74A or PUMA-G) as a new therapeutic target. Trends Pharmacol Sci. 2006;27:384-90.

48. Taggart AK, Kero J, Gan X, Cai T-Q, Cheng K, Ippolito M, et al. (D)- $\beta$-hydroxybutyrate inhibits adipocyte lipolysis via the nicotinic acid receptor PUMA-G. J Biol Chem. 2005;280:26649-52.

49. Zandi-Nejad K, Takakura A, Jurewicz M, Chandraker AK, Offermanns S, Mount D, et al. The role of HCA2 (GPR109A) in regulating macrophage function. FASEB J. 2013;27:4366-74.

50. Digby JE, Martinez F, Jefferson A, Ruparelia N, Chai J, Wamil M, et al. Anti-inflammatory effects of nicotinic acid in human monocytes are mediated by GPR109A dependent mechanisms. Arterioscler Thromb Vasc Biol. 2012;32:669-76.

51. Kulms D, Schwarz T. NF-kappaB and cytokines. Vitam Horm. 2006;74:283-300.

\section{Submit your next manuscript to BioMed Central and take full advantage of:}

- Convenient online submission

- Thorough peer review

- No space constraints or color figure charges

- Immediate publication on acceptance

- Inclusion in PubMed, CAS, Scopus and Google Scholar

- Research which is freely available for redistribution 